\title{
World Rabies Day: a prime role for veterinarians in rabies control
}

\section{Mohan S. Maddur, Srini V. Kaveri and Jagadeesh Bayry}

We read with interest the recent editorial (Biting back against rabies. Nature Rev. Microbiol. 8, $676(2010))^{1}$ that was published just in time for World Rabies Day and summarized the current situation and feasible control measures. Rabies is a deadly viral disease of warm-blooded animals and has zoonotic importance ${ }^{2}$. Unlike other infectious diseases, rabies is transmitted to humans through a bite or scratch smeared with saliva containing the virus, so limiting contact with affected animals has a crucial role in disease prevention. The disease is endemic in developing countries in Asia, Africa and South America and contributes substantially to human deaths due to infections, particularly in rural areas. Humans are considered to be the dead-end host and, hence, animals serve as a reservoir pool for maintenance and amplification of the virus. Therefore, veterinarians occupy prime roles for control of the disease.
Veterinarians are responsible for diagnosing the disease in a wide range of animals, including domestic animals (such as cattle and buffaloes), pets (such as dogs and cats) and wild animals (such as raccoons, skunks, foxes and bats), all of which are potential sources of the virus for humans ${ }^{3}$. In most developing countries, there is direct contact between domestic animals and pets, which makes regular vaccination of these animals by veterinarians crucial for the prevention of rabies in the human population. Furthermore, the presentation of rabies in animals in two distinct forms, the furious form and the dumb form, makes detection of the disease much more complicated. Thus, proper diagnosis of the rabid animal by a veterinarian is a must to remove the source of this deadly disease.

Dogs serve as a major source of rabies virus for disease transmission and, hence, control measures should also target this species of animal ${ }^{4}$. Although annual vaccination of dogs, including street dogs, is important, control of the dog population is also crucial to prevent disease spread. For multiple reasons, it is not feasible to completely eliminate the street-dog population in many countries. Hence, animal birth control programmes are implemented to control the street-dog population, and so another role for veterinarians is in reducing the size of the host population for rabies virus.

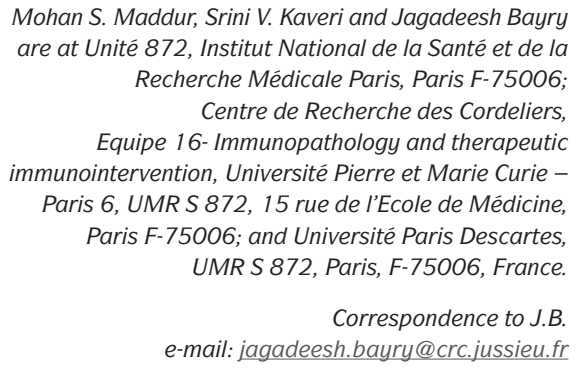
Recherche Médicale Paris, Paris F-75006, Centre de Recherche des Cordeliers, Equipe 16-Immunopathology and therapeutic immunointervention, Université Pierre et Marie Curie Paris 6, UMR S 872, 15 rue de l'Ecole de Médicine, Paris F-75006; and Université Paris Descartes, UMR S 872, Paris, F-75006, France.

Correspondence to J.B.

e-mail: jagadeesh.bayry@crc.jussieu.fr

1. No authors listed. Biting back against rabies. Nature Rev. Microbiol. 8, 676 (2010).

2. Schnell, M. J., McGettigan, J. P., Wirblich, C. \& Papaneri, A. The cell biology of rabies virus: using stealth to reach the brain. Nature Rev. Microbiol. 8 , 51-61 (2010).

3. Haider, S. Rabies: old disease, new challenges. Can. Med. Assoc. J. 178, 562-563 (2008).

4. Voelker, R. Global effort takes aim at rabies. JAMA 298, 1749-1750 (2007).

Competing interests statement

The authors declare no competing financial interests. 